\title{
AUC Over Dosing Interval Normalized by Weight
}

National Cancer Institute

\section{Source}

National Cancer Institute. AUC Over Dosing Interval Normalized by Weight. NCI

Thesaurus. Code C92325.

The area under the curve (AUC) for the defined interval between doses (TAU) divided by the weight. 